\title{
PIV Measurements of Flow in a Centrifugal Blood Pump: Steady Flow
}

Magnetically suspended left ventricular assist devices have only one moving part, the impeller. The impeller has absolutely no contact with any of the fixed parts, thus greatly reducing the regions of stagnant or high shear stress that surround a mechanical or fluid bearing. Measurements of the mean flow patterns as well as viscous and turbulent (Reynolds) stresses were made in a shaft-driven prototype of a magnetically suspended centrifugal blood pump at several constant flow rates $(3-9 \mathrm{~L} / \mathrm{min})$ using particle image velocimetry (PIV). The chosen range of flow rates is representative of the range over which the pump may operate while implanted. Measurements on a three-dimensional measurement grid within several regions of the pump, including the inlet, blade passage, exit volute, and diffuser are reported. The measurements are used to identify regions of potential blood damage due to high shear stress and/or stagnation of the blood, both of which have been associated with blood damage within artificial heart valves and diaphragm-type pumps. Levels of turbulence intensity and Reynolds stresses that are comparable to those in artificial heart valves are reported. At the design flow rate (6 L/min), the flow is generally well behaved (no recirculation or stagnant flow) and stress levels are below levels that would be expected to contribute to hemolysis or thrombosis. The flow at both high $(9 \mathrm{~L} / \mathrm{min})$ and low $(3 \mathrm{~L} / \mathrm{min})$ flow rates introduces anomalies into the flow, such as recirculation, stagnation, and high stress regions. Levels of viscous and Reynolds shear stresses everywhere within the pump are below reported threshold values for damage to red cells over the entire range of flow rates investigated; however, at both high and low flow rate conditions, the flow field may promote activation of the clotting cascade due to regions of elevated shear stress adjacent to separated or stagnant flow. [DOI: 10.1115/1.1865189]

\section{Introduction}

A left ventricular assist device (LVAD) can effectively relieve some strain on a native heart that has been weakened by disease or damage, and allow sufficient blood flow to maintain normal physiologic function. The LVAD inlet is normally attached to the left ventricle and the pump output rejoins the native flow at the aorta. The clinical effectiveness of LVADs has been demonstrated; however, all of the currently available pumps are used only as a "bridge to transplant" because of the damage that they cause to blood and/or their limited mechanical design life.

Prior studies of both artificial heart valves and diaphragm-type pumps have related fluid dynamics to blood damage, including both hemolysis (red blood cell damage) and thrombosis (clotting). Hemolysis is associated with the exposure of blood to high shear stresses that causes the tearing of red blood cell membranes. Shear stress is an unavoidable property of viscous fluid flow, but can be minimized by a careful pump design that controls the levels of shear stress and the exposure time of blood cells to high-stress regions. Mathematical models to quantify hemolysis as a function of time and shear stress have been developed [1,2]. These models may be used in conjunction with a measured velocity field to quantitatively evaluate the potential for hemolysis within a pump.

Thrombosis consists of a complex series of chemical and mechanical reactions that leads to the formation of a blood clot (thrombus). The clotting cascade is initiated by the activation of platelets and concludes with the adherence of platelets and leuko-

${ }^{1}$ Corresponding author. Current address: Section of Evolution \& Ecology, University of California, One Shields Avenue, Davis, CA 95616.

Contributed by the Bionengineering Division for publication In the Journal OF BiomeChanical EngineERING. Manuscript received October 7, 2003. Final manuscript received September 20, 2004. Associate Editor: C. Ross Ethier. cytes to the endothelium (in native vessel tissue), to a foreign substance (in the case of a prosthetic), or the formation of bloodborne thromboemboli. Thrombosis is dependent on surface material properties, but it is also related to fluid dynamics. It has been shown that regions of stasis (stagnant flow) and low shear at the blood/material interface are more prone to thrombus formation, particularly if these regions are located immediately after a region of high shear stress (which may mechanically activate platelets) $[3,4]$. Thrombosis, like hemolysis, is a function of the detailed fluid structure within the pump. Attached thrombi are not necessarily harmful, but when thrombi become detached from the material surface (thromboembolism) or grow so large that they burst and release material into the blood stream (thrombolysis), the resulting embolisms can have serious consequences. Thrombus formation and subsequent embolism is perhaps the most difficult obstacle to long-term artificial circulatory support [5].

Current state-of-the-art LVADs are magnetically suspended rotary pumps. They have only one moving part (the pump impeller), which has no contact with any fixed parts. This increases design life over diaphragm-type pumps by eliminating wear and the inclusion of flexible materials, which tend to become brittle and fail with age. Further, these pumps are capable of greatly reducing the regions of stagnant or high shear flow that surround a mechanical or fluid bearing. Because both hemolysis and thrombosis are related to fluid dynamics, understanding the fluid behavior inside these new rotary pumps is critical. To date, however, only limited quantitative measurements of the flow field within rotary-type magnetically suspended LVADs have been made.

Several published reports of qualitative flow visualization [6] and quantitative velocity measurements in industrial-sized rotary pumps are found in the literature. Quantitative measurements of velocity in industrial-sized pumps include studies using laser Dop- 


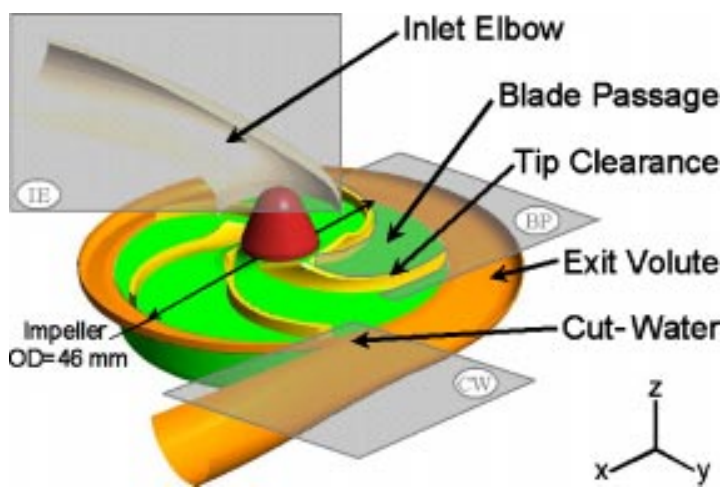

Fig. 1 Cutaway view of the pump showing regions of interest and location of laser sheets that define the measurement planes for three-measurement configurations. Flow enters the inlet cannula from the left ventricle and passes into the blade passages. Fluid exiting the impeller is collected in the exit volute and flows past the cutwater to the exit of the pump. Measurement planes labeled as IE, inlet elbow; BP, blade passage; and $\mathrm{CW}$, cutwater.

pler velocimetry (LDV) at both design [7] and off-design points of the flow within the blade passage [8-10] and exit volute [11]. Particle image velocimetry (PIV) has been used for quantitative measurements of steady flow within the inlet [12] and the exit volute [13] of an industrial-sized centrifugal pump and used to investigate the effect of varying the shape of the cutwater [14] in one study.

Qualitative [15,16] and quantitative LDV [17] and PIV techniques have been used to measure the flow within the pumping chamber [18] and in the inlet and outlet regions [19,20] of diaphragm (not rotary) LVADs. Qualitative flow visualization has been used frequently in the study of miniaturized rotary blood pumps of both centrifugal [21-23] and axial [24] configuration. Further, LDV has been used in rotary blood pumps in some instances [25], and PIV has been used for quantitative measurements in the outlet cannula of rotary-type pumps [26] and in the clearance region of the previous generation of the pump used in this study [27]. In one study, particle tracking velocimetry (PTV), a quantitative technique closely related to PIV, was used to characterize the flow within all regions of a scale model of a miniature pump at one operating condition [28].

To date, published quantitative data regarding the details of the flow internal to rotary LVADs are limited in one of two ways. First, due to geometric and optical constraints, they do not include measurement within the blade passage. Secondly, they are limited to a single operating condition and are, therefore, not representative of the range of flow rates under which a rotary pump will operate when implanted.

In the current study, PIV was used to characterize the flow within a centrifugal LVAD under steady flow conditions. The measurements presented herein consist of the mean velocity field and turbulence statistics over the range of flow rates the pump is likely to experience when implanted. Measurements are reported in the inlet, blade passage, and exit volute. Also included is a prediction of the effects that specific flow features will have on blood damage.

\section{Experimental Methods}

LVAD and Flow Loop. The LVAD geometry investigated during these experiments was the second iteration of the fourth generation (CF4b) HeartQuest ${ }^{\mathrm{TM}}$ pump [29]. Pumps with identical internal flow paths have been used in animal implant tests and modeled extensively with computational fluid dynamics (CFD) [30]. The blood-wetted flow paths of the pump are shown in a cutaway view in Fig. 1. In order to accomplish PIV measurements within the LVAD, a prototype pump that allowed for optical access into the interior of the pump was built, as described elsewhere [31]. This pump was mounted on a shaft instead of suspended by magnetic bearings. This allowed control over the impeller position and construction of this test rig before the magnetic suspension system was completed. The impeller shaft had three small "teeth" located on the outer diameter that formed an interference fit with the inner diameter (ID) of the impeller. The teeth were $3 \mathrm{~mm}$ tall (axial direction) and obstructed less than $20 \%$ of the cross-sectional area of the back clearance gap over their axial height. Prior to these experiments, CFD simulations of the flow in the back clearance gap with and without these teeth were compared to confirm that the teeth did not affect the flow in the back clearance or in other regions of the pump.

The pump was part of a closed-loop system that allowed the flow loop resistance and pump rotational speed to be controlled. The pump flow rate and pressure rise were functions of the resistance and rotational speed. Details of the flow loop and measurements of the overall pump performance have been presented elsewhere [32]. A LabView (National Instruments, Austin, TX) program displayed and recorded data measured from the flow loop, including: LVAD flow rate, ventricle flow rate, left ventricular pressure, aortic pressure, venous pressure, working fluid temperature, and rotational speed. LabView was also used to control the motor speed via a simple closed-loop feedback controller that maintained the pump operating speed to within $\pm 1 \%$ of the requested shaft speed.

Fluid was delivered to the inlet elbow of the pump through a small reservoir and a straight inlet cannula $(200 \mathrm{~mm} \times$ $12.5 \mathrm{mmID})$. The angle between the inlet cannula and the $y$-axis was maintained at 25 degrees. In the implanted condition, the cannula is bent to fit the particular patient anatomy. This use of a straight cannula in the experiments provided a simple geometry upstream of the pump needed to create a reproducible inflow boundary condition for comparison to CFD and future experiments. The ID of the cannula was tapered to provide a smooth transition to the inlet elbow of the pump (12 mm ID). The cannula inlet was a reservoir of approximately the same volume as an adult left ventricle and the relative position of the inlet cannula in the simulated ventricle is the same as it is when implanted.

Selection of Operating Conditions. Because of the way in which the pump is attached to the human circulatory system when implanted, the flow through the pump varies with the pulsing of the native ventricle $[33,34]$. Based on measurements of the global pump performance, a speed of $2100 \mathrm{rpm}$ was selected as the nominal operating speed. When operating with a beating heart of weak strength at this rotational speed, the pump delivers a timeaveraged flow of $6 \mathrm{~L} / \mathrm{min}$ with instantaneous flow rates varying from 3-9 L/min during each heartbeat. Because the pump operates over such a wide range of operating conditions during every heartbeat, the flow field at operating points other than the nominal flow rate of $6 \mathrm{~L} / \mathrm{min}$ was investigated. All of the measurements presented herein are for steady flow rate through the LVAD; measurements of the fluid dynamics while operating with a pulsing ventricle are presented elsewhere [34]. The pump was run at a constant speed of 2100 during all experiments. Between experiments, the systemic resistance was adjusted to vary the flow rate from $3 \mathrm{~L} / \mathrm{min}$ to $9 \mathrm{~L} / \mathrm{min}$.

Particle Image Velocimetry. PIV is a technique that measures the instantaneous velocity field within an illuminated plane of the fluid field using light scattered or fluoresced from particles seeded into the fluid [35]. It differs from techniques called "flow visualization" in that PIV is quantitative. PIV measures the instantaneous two-dimensional velocity field illuminated by the laser sheet, unlike LDV, which is a single-point measurement. PIV has recently matured to a reliable technique that is used in a wide variety of applications [36].

A Kodak digital CCD camera, PIVCAM 10-30, was used for 
image acquisition. The camera is a $1008 \times 1008$ pixel CCD with 8-bit resolution designed for very fast acquisition of two successive frames. For these experiments, the time separation varied from 75 to $150 \mu \mathrm{s}$, depending on the speed of the flow and camera field of view. The laser and camera were synchronized by software and hardware sold by TSI, Inc. A dual-cavity pulsed Nd:Yag laser (Spectra Physics, Mountain View, CA) with frequencydoubling crystal illuminated polymer particles (Duke Scientific, Palo Alto, CA) that are added to the flow. These particles are nominally $10 \mu \mathrm{m}$ in diameter and are filled with a fluorescent dye that is necessary to discriminate between the light fluoresced by the particles and that scattered from surfaces of the pump [31]. The seed particles have a specific gravity of 1.05 , which is a very good match to the working fluid $(\mathrm{SG}=1.05)$, and ensured good particle tracking [37]. The delay and jitter between the $Q$-switch trigger and light emission is $<5 \mathrm{~ns}$, so laser timing is not a significant source of error.

Using a fluid with the same index of refraction as the surrounding material is known as "index matching" and is critical to minimize refraction when making quantitative measurements inside small regions with complex curvature. Aqueous salt solutions are often used for index matching of the fluid with the pump housing material [38,39]; however, the high concentrations of salt result in fluid densities higher than blood so that they do not effectively model blood flow through a centrifugal pump [32]. Summaries of index matching fluids included in Durst [40] and Budwig [41] do not include any fluids that match the required refractive index of the pump housing and the density and viscosity of blood. Dibutyl phthalate was selected as a working fluid for the current experiments because it meets all of these criteria. At $50^{\circ} \mathrm{C}$, the liquid has a viscosity of $5 \mathrm{cp}$ and a specific gravity of 1.05 (compared to 3-5 $\mathrm{cp}$ and $\mathrm{SG}=1.05$ for human blood at normal hematocrit levels [42]) and an index of refraction of 1.485 (as compared to 1.492 for the housing acrylic) for the wavelength used $(532 \mathrm{~nm})$. The working fluid was maintained at $50^{\circ} \mathrm{C} \pm 2{ }^{\circ} \mathrm{C}$ by means of a copper-tubing heat exchanger.

The movement of the impeller blades with respect to fixed geometries such as the cut-water creates the potential for timevarying flows within the exit volute of the pump. In order to remove the effects of the rotor/stator interaction due to the blade passage, measurements within the blade passage and exit volute were made synchronously with respect to the impeller position. A pulse generator (Stanford Research Systems, Palo Alto, CA) was used to create a controlled delay between the shaft encoder and the PIV system, effectively "freezing" the impeller at any given position.

The position and orientation of the laser sheets (and, therefore, measurement planes) are shown in Fig. 1. Three configurations were used to resolve the flow in critical regions of the pump. These are referred to as the inlet elbow, blade passage, and cutwater configurations. In each configuration, the laser sheet was translated normal to the sheet to resolve the two components of measured velocity on a three-dimensional measurement grid.

For the data reduction, an adaptive mesh cross-correlation algorithm created by Scarano and Riethmuller was used to calculate velocities from image pairs [43]. The algorithm returned a twodimensional (2D) grid of two components of measured velocity for each image pair that was processed. In addition, it recorded a signal-to-noise ratio for each measured velocity vector so that criteria could be applied to insure that only quality measurements were used. During processing, a digital mask was applied to discriminate between areas of the image that are fluid versus solid. The definition of this boundary was straightforward because the intensity of the fluid containing fluorescent particles is much higher than that of the solid material.

All measurements for this work used an initial interrogation window size of $64 \times 64$ pixels and two refinement steps. Replacement of nonvalid vectors and some smoothing was used for the intermediate steps. The program applies a weighted 2D moving average to the intermediate velocity fields. The final image interrogation used $16 \times 16$ pixel interrogation windows with a $50 \%$ overlap, so that the measurement grid spacing was 8 pixels, corresponding to 0.3 to $0.4 \mathrm{~mm}$ for the camera field of view used in these experiments. The original image size is $1008 \times 1008$ pixel, resulting in an $i, j$ ordered grid that is $124 \times 124$. Vectors with a signal-to-noise ratio of less than 3.0 were removed, without replacement. No smoothing was applied to the final velocity field.

The series of instantaneous velocity fields was processed to determine statistical quantities according to the equations included herein. The instantaneous velocities $\tilde{u}$ of a turbulent flow can be expressed, according to the Reynolds decomposition, as the summation of a steady mean velocity $U$ and a fluctuating velocity $u$. The magnitude of the mean $U$ and standard deviation $u^{\prime}$ of the instantaneous velocity $\tilde{u}$ are statistical descriptions of the flow and can be found from a set of instantaneous velocity measurements, each made at discreet times, by ensemble averaging [44]. The standard deviation $u^{\prime}$ of the mean velocity $U$ is exactly equal to the root mean square (rms) of the fluctuating component of velocity $u$. Fluctuations caused by turbulence and other random or nonrandom processes contribute to the standard deviation $u^{\prime}$. Without being able to resolve the frequency spectrum of these fluctuations, it is incorrect to characterize these fluctuations as turbulence, so that $u^{\prime}$ is referred to as rms of fluctuating velocity or simply rms throughout.

The Reynolds stress is a symmetric, second-order tensor (6 independent terms, 3 normal stresses, and 3 shear stresses), of which two normal stresses and one Reynolds shear stress may be calculated from simultaneous measurements of two components of velocity. The magnitude of Reynolds shear stresses is dependent on the coordinate system used to evaluate them. True values of the maximum stresses in the flow, which are particularly important when comparing to values reported to cause blood damage, were calculated according to the method described by Baldwin [38]. These are referred to as the "major Reynolds normal stress" (MRNS) and "major Reynolds shear stress" (MRSS) and are defined as the stresses along the principal axes of the stress tensor. Although the full tensor is three-dimensional, the principal axes and corresponding principal shear stress within the twodimensional measurement plane were calculated with the understanding that these were equal to or less than the true value of the three-dimensional principal stress.

Derivative quantities such as the viscous (molecular) shear stress and the vorticity of the flow field may also be calculated from the spatial derivatives of the velocity field. One component of vorticity and two components of shear stress within the measurement plane are found from spatial derivatives of either the mean or instantaneous flow field.

As the number of samples $N$ approaches infinity, the value of the ensemble average will converge to exactly that of the true mean of the velocity, $U$, so long as some conditions about the statistical nature of the flow are met [45]. For all measurements shown, 200 instantaneous vector fields $(N=200)$ were processed to determine the statistics. A convergence study, which was completed to determine the effect of this limited sample size, led to an uncertainty of $\pm 3 \%$ mean speed, $\pm 10 \% \mathrm{rms}$, and $\pm 15 \%$ MRNS or MRSS [46].

\section{Results}

Inlet. The mean velocity, $U$, within the inlet is shown in Fig. 2 for flow rates of 3,6 , and $9 \mathrm{~L} / \mathrm{min}$ along with streamlines. The mean velocity field shows no indications of recirculation or stagnation within the inlet elbow over the range of flow rates shown, 3-9 L/min, with one exception. This is a small region at the nose of the outer curvature of the inlet elbow where the velocities are very low and moving antegrade to the mean flow very near the wall. This region is evident in the streamlines within the elbow center plane (left side of Fig. 2) and also in other planes of con- 

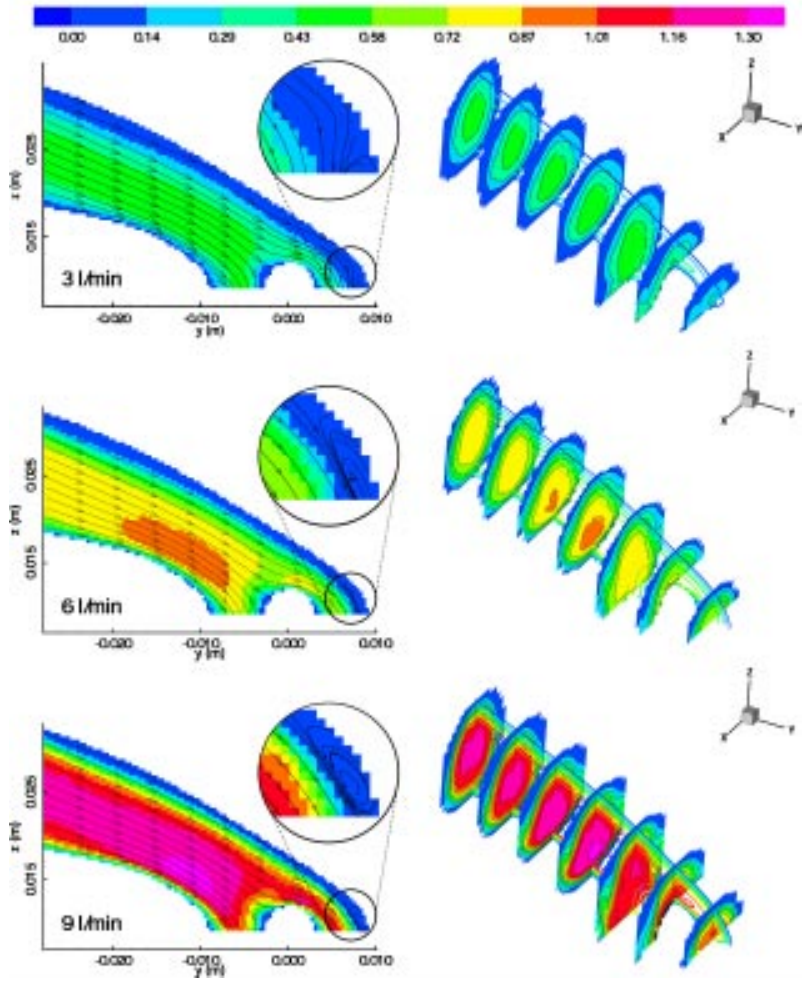

Fig. 2 Distribution of mean velocity within the inlet elbow for flow rates of 3,6 , and $9 \mathrm{~L} / \mathrm{min}$. One plane of constant $x$, at inlet center plane (left), and several planes of constant $y$ (right) are shown. Labels on the axes are the exact distance from a common origin located on the axis of rotation of the impeller. The entire square representing that particular grid point is colored a constant shade, resulting in the appearance of pixels in the plot. The pixels are the size of the resolution of the measurement.

stant $x$ (not shown). The spindle that protrudes up into the inlet elbow evenly divides the flow. The flow is symmetrical with respect to the geometrical plane of symmetry, as is expected. The mean velocity distribution is highest toward the inner curvature of the inlet elbow at all flow rates.

Levels of the rms of fluctuating velocity are significant (as high as $20 \%$ of the local mean velocity and $15 \%$ of the maximum mean velocity in the inlet elbow) in a region located midway between the centerline and outer walls, as is shown in Fig. 3. This is a cylindrically shaped region of high levels of rms near the inlet to the inlet elbow and evolves into a horseshoe shape (as viewed in the planes of constant $y$ ) midway through the inlet elbow. High levels of rms generally occur in areas of gradients of mean velocity, but are not immediately adjacent to the walls. The locations of high levels of rms of fluctuating velocity were the same for all flow rates, with the magnitudes increasing with flow rate.

Although regions of highest rms coincide with regions of highest MRSS, Fig. 3, the magnitude of rms is not directly proportional to magnitudes of MRSS. For example, the flow field at $9 \mathrm{~L} / \mathrm{min}$ has an rms of fluctuating speed in the range of $0.17 \mathrm{~m} / \mathrm{sec}$ near both the inner and outer curvatures. Only a small subset of this area, however, has MRSS levels as high as $35 \mathrm{~Pa}$, whereas the majority of this area has MRSS levels of about $10 \mathrm{~Pa}$.

Everywhere within the pump, the magnitude of viscous shear, as determined from velocity gradients in the measurement plane, is much lower in value than the magnitude of Reynolds stresses (indicating that this is a flow dominated by turbulent momentum transfer). Values of viscous shear are highest in the boundary layer along the inner and outer curvatures of the inlet elbow. The mag-
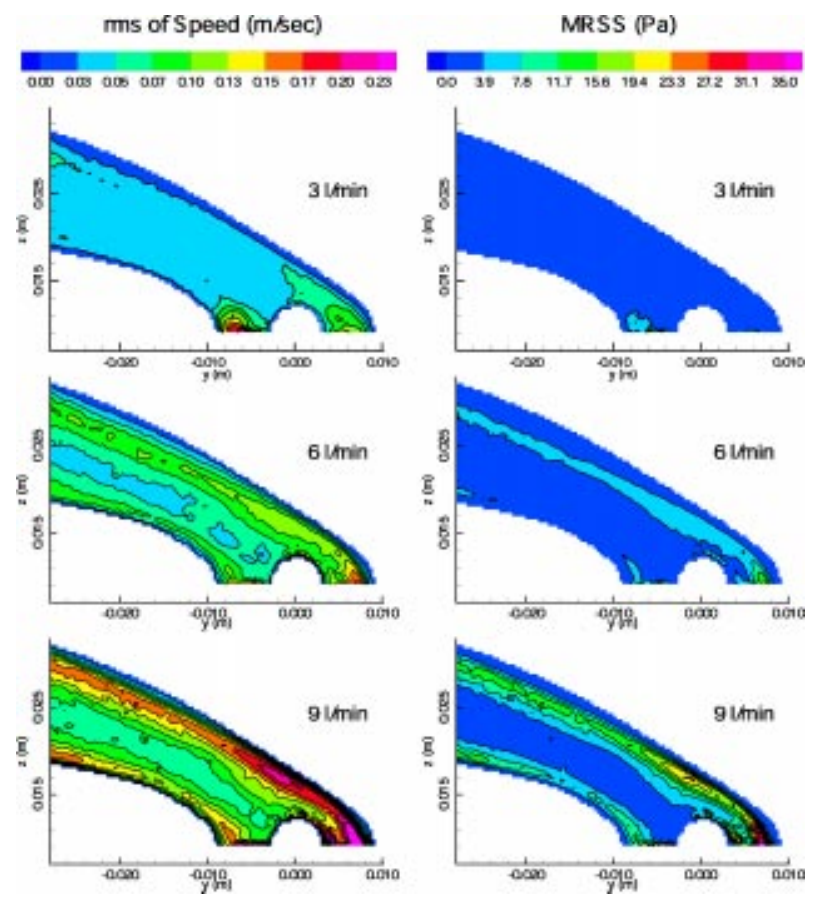

Fig. 3 Distribution of rms of velocity (left) and MRSS (right) within the inlet elbow for flow rates of 3,6 , and $9 \mathrm{~L} / \mathrm{min}$ at inlet center plane

nitude of viscous shearing along the inlet walls increases with flow rate and has a maximum value of $5 \mathrm{~Pa}$ along the inner wall at a flow rate of $9 \mathrm{~L} / \mathrm{min}$.

The fluctuating component of the velocity field $u$ at an instant in time shows the unsteady structures within the flow that are larger than the measurement technique's spatial resolution. This is achieved by subtracting the mean field $U_{i, j}$ from the instantaneous velocity field $\tilde{u}_{i, j}$ Two examples of the instantaneous velocity field (at top and labeled $a$ and $b$ ) are shown along with the fluctuating component corresponding to each (bottom) at the inlet elbow center plane in Fig. 4. These measurements correspond to separate instants in time and were selected because they represent the two extremes (one with a clearly defined clockwise or counterclockwise circulation) of the velocity fluctuations in this region. Contours of speed for both fields are included to help reveal structures within the flow. The instantaneous velocity field (Fig. 4(a)) has a
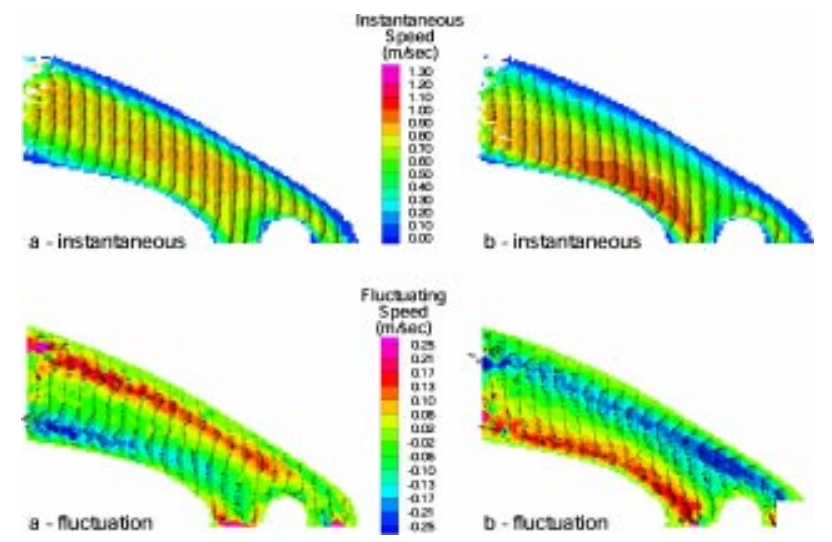

Fig. 4 Examples of instantaneous $\widetilde{u}$ (top) and fluctuation $u$ (bottom) velocity field in the inlet elbow at steady flow rate of $6 \mathrm{~L} / \mathrm{min}$ taken at two different instants in time. Contours of speed (magnitude of the instantaneous or fluctuation velocity). 

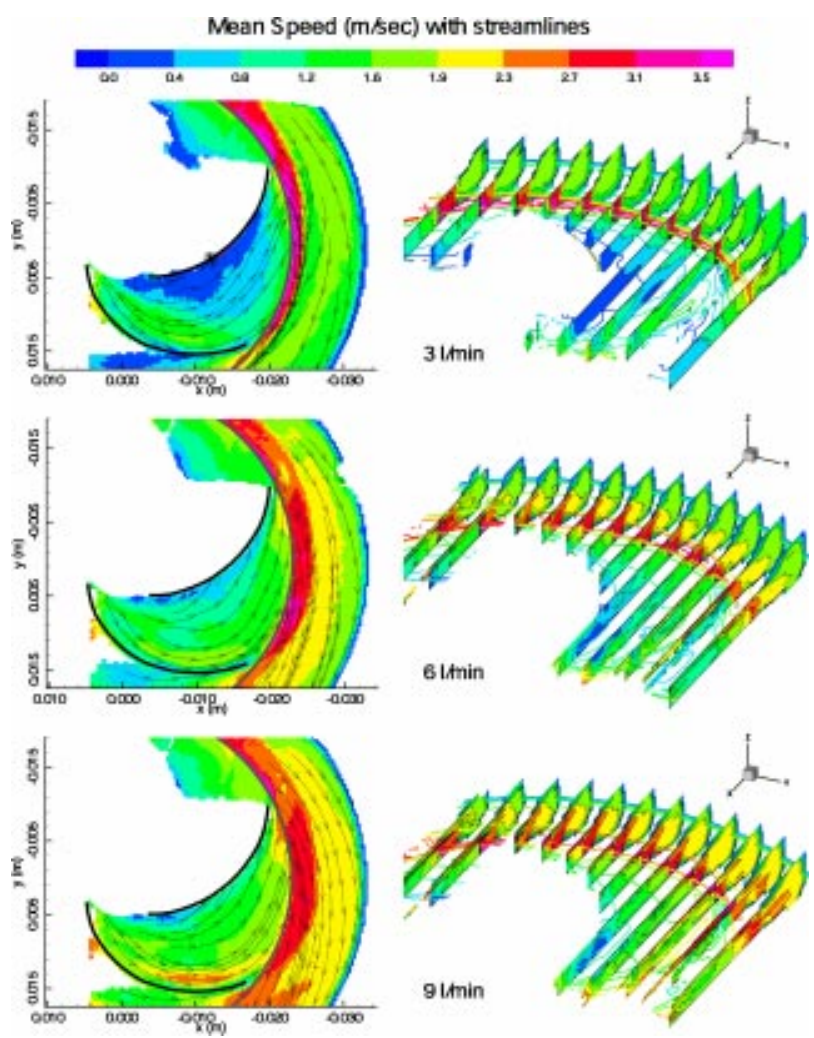

Fig. 5 Distribution of mean velocity within the blade passage for flow rates of 3,6 , and $9 \mathrm{~L} / \mathrm{min}$. One plane of constant $z$ at midblade height (left) and several planes of constant $y$ are shown. Location of the two blades defining the blade passage are shown as black lines. The location of the impeller OD is shown as a grey line. Blade rotation is clockwise, so pressure side of blade is just below upper blade and suction side is just above lower blade. Within the blade passage, velocity is relative to the impeller rotation. For all radii greater than impeller OD, absolute velocity is shown.

higher than mean velocity along the outer curvature of the inlet elbow and lower than mean velocity along the inner curvature. For field $b$, positions of high and low velocity are reversed. This "flip-flopping" of the high velocity region to one surface or the other is representative of the fluctuations in the inlet elbow of the pump.

It can be seen from the figure that the fluctuations within the inlet elbow are very large compared to the size of the inlet elbow. The magnitude of fluctuations is as large as $10 \%$ of the mean velocity $(0.15 \mathrm{~m} / \mathrm{s})$, which agrees with values of the rms of fluctuating speed, presented in Fig. 3. There is no coherence from one instantaneous measurement to the next, meaning that the frequency of fluctuations is higher than $10 \mathrm{~Hz}$, the sampling rate. This corresponds to structures smaller than $\sim 10 \mathrm{~cm}$ (at $1 \mathrm{~m} / \mathrm{s}$ mean velocity), which is much larger than the diameter of the inlet elbow $(1.25 \mathrm{~cm})$, but similar to the length of the inlet cannula $(20$ $\mathrm{cm})$. Based on this and the spatial distributions of fluctuation velocity given in Fig. 4, the nominal size of the fluctuations may be estimated as being between 1 and $10 \mathrm{~cm}$.

Blade Passage. The mean velocity within the blade passage is shown in Fig. 5 for flow rates of 3, 6, and $9 \mathrm{~L} / \mathrm{min}$. For all measurements within the blade passage, the velocity relative to the impeller rotation is shown because this more clearly shows fluid patterns relative to the blades and potential recirculation in this region. For all radii greater than the outer diameter (OD) of the impeller, the absolute velocity is shown. The location of the impeller OD, shown as a grey line in Fig. 5, also reveals itself by
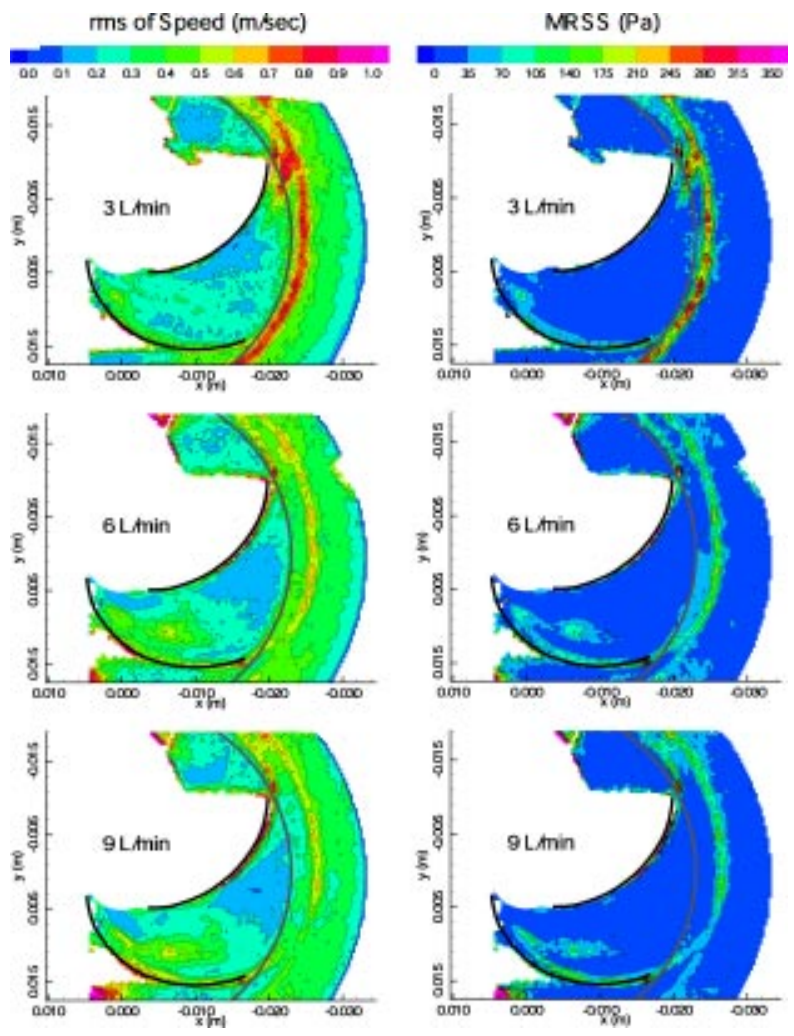

Fig. 6 Distribution of rms of velocity (left) and MRSS (right) within the blade passage for flow rates of 3,6 , and $9 \mathrm{~L} / \mathrm{min}$ at midblade height

a sudden change in the direction of fluid streamlines. In reality, the fluid does not make a sudden change in direction here; this high curvature in streamlines and discontinuity in measured speed are artifacts of the interface of the rotating and absolute reference frames selected to display the data. While this transformation of reference frames affects the mean velocity field, it has no effect on the rms and turbulent statistics and a negligible effect on the measurements of viscous shear based on gradients in the flow, except at exactly the impeller OD.

The relative mean flow within the blade passage generally moves radially outward and follows the contour of the blades. The mean velocity field shows a low velocity region on the pressure side of the blade for all flow rates tested. At the lowest flow rate, $3 \mathrm{~L} / \mathrm{min}$, the flow is directed inward radially, creating a recirculation region within the blade passage. Similarly, there is a high velocity region on the suction side of the blade at all flow rates, as is typical for centrifugal pumps [47]. The absolute mean speed within the exit volute is highest just at the exit of the blade, but is fairly uniform throughout the volute.

Levels of the rms of fluctuating velocity (Fig. 6) within the blade passage are substantial $(0.1-0.6 \mathrm{~m} / \mathrm{s})$. The highest levels of rms are seen in a region located on the suction side of the blade for all flow rates. At 6 and $9 \mathrm{~L} / \mathrm{min} \mathrm{rms}$ is $\sim 0.5 \mathrm{~m} / \mathrm{s}$, which corresponds to $40 \%$ of the local relative fluid velocity, $20 \%$ of the local absolute velocity, and $15 \%$ of the maximum absolute velocity seen in the blade passage.

Within the exit volute, the rms of fluctuating velocity is highest within a clearly defined shear layer between the fluid exiting the impeller and fluid within the volute. The angle and depth of penetration of this layer from the trailing edge of the blades into the volute depends on the flow rate, with both angle and depth of penetration increasing with flow rate. At low flow rates, levels of rms within this layer are as high as $0.9 \mathrm{~m} / \mathrm{s}$, which is approximately $30 \%$ of the local mean. 

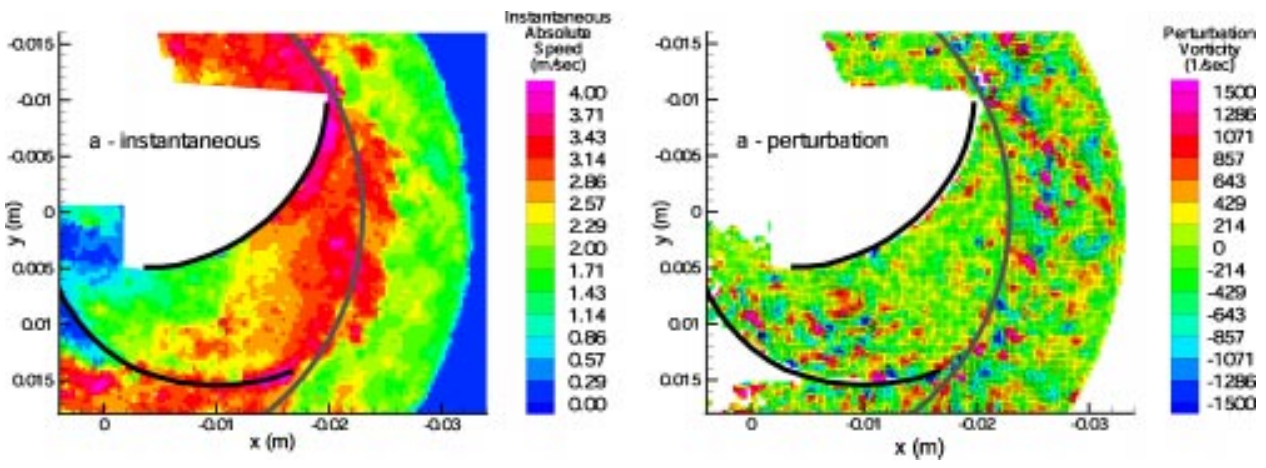

Fig. 7 Example of instantaneous $\widetilde{u}$ (left) and fluctuation $u$ (right) velocity field for the blade configuration at $6 \mathrm{~L} / \mathrm{min}$ at the midblade height with contours of vorticity shown for the fluctuation velocity field

The distributions of MRSS is similar to the distribution of rms; however, the levels of MRSS located in the shear layer between the fluid exiting the impeller and the fluid in the exit volute are higher than those within the blade passage for all flow rates (Fig. 6). Levels of MRSS are highest for the lowest flow rates because as the flow rate decreases, absolute velocity within the impeller increases and absolute velocity within the volute decreases, increasing shear at the impeller exit. The shape of the shear layer clearly locates the position of the blade wake and penetration of the fluid exiting the blade passage into the exit volute flow.

As was the case for the inlet, levels of viscous shear associated with velocity gradients in the measurement plane are everywhere much lower value than Reynolds stresses. Values of viscous shear are most significant along the outer wall of the exit volute and within the mixing layer just outside of the impeller exit (maximum value of approximately $15 \mathrm{~Pa}$ ). The magnitude of viscous stresses along the volute wall increases with flow rate. The magnitude of viscous shear in the mixing layer decreases as a function of flow rate and also has the identical distribution as the Reynolds stress in this region.

An example of the instantaneous velocity field (left) within the blade passage along with the fluctuating component (right) at the same instant in time is shown in Fig. 7. Contours of absolute speed (this measurement is made before calculating the velocity relative to the impeller) are given for the instantaneous velocity field. The fluctuating velocity field includes contours of vorticity instead of speed, which helps to reveal structures within the flow because a distinct eddy of any given size shows up as a region of either high or low vorticity. The structures revealed within the flow by this method represent the vorticity of the fluctuation velocity at a single instant in time.

The fluctuations within the blade passage and exit volute are much smaller in size than those in the inlet elbow. The size of the largest structures, 2-3 mm, is approximately the same as the blade height. There are no large-scale fluctuations within the blade passage similar to those that were seen in the inlet elbow at any flow rate. The precise location of the structures is random and not correlated from one instantaneous measurement to the next. The general location, however, is correlated with the location of the highest values of rms of fluctuating velocity shown in Fig. 6. In this region of the pump, small-scale fluctuations contribute to rms. This size structure and the random distribution are more representative of traditional turbulence.

Cutwater. At the design flow rate $(6 \mathrm{~L} / \mathrm{min})$, fluid streamlines impact the cutwater with a very small incidence angle, as is the design goal (Fig. 8). At lower than design flow rates, the streamlines are moving inward radially as they contact the cutwater; at higher than design flow rates, the streamlines move outward radially. For all measured flow rates, the velocity distribution in the throat is skewed toward the outer radius. This continues into the exit diffuser of the pump, where there is a high velocity region on the outer wall and a low velocity region on the inner wall. At the highest flow rate presented, $9 \mathrm{~L} / \mathrm{min}$, the flow is separated and a recirculation region exists within the exit diffuser. The threedimensional distribution of velocity also reveals the spiral flow (outward radially near the center and inward radially near the walls) in the exit volute, which is common to volute flows, but contributes to losses [47].

Levels of the rms of fluctuating velocity, Fig. 9, are significant. The locations of high levels of rms are in the region just downstream of the cutwater. This is most pronounced at the highest flow rates, when fluid streamlines are moving radially outward and high levels of $\mathrm{rms}(0.7 \mathrm{~m} / \mathrm{s}, 30 \%$ of the local mean velocity $)$ exist in the wake of the cutwater. At low flow rates, levels of high rms are located inside the cutwater, due to the streamlines flowing inward radially. In addition to these wakes, there are measurable and fairly uniform levels of rms throughout all of the flow in this region. The magnitude of this background levels of rms were pro-

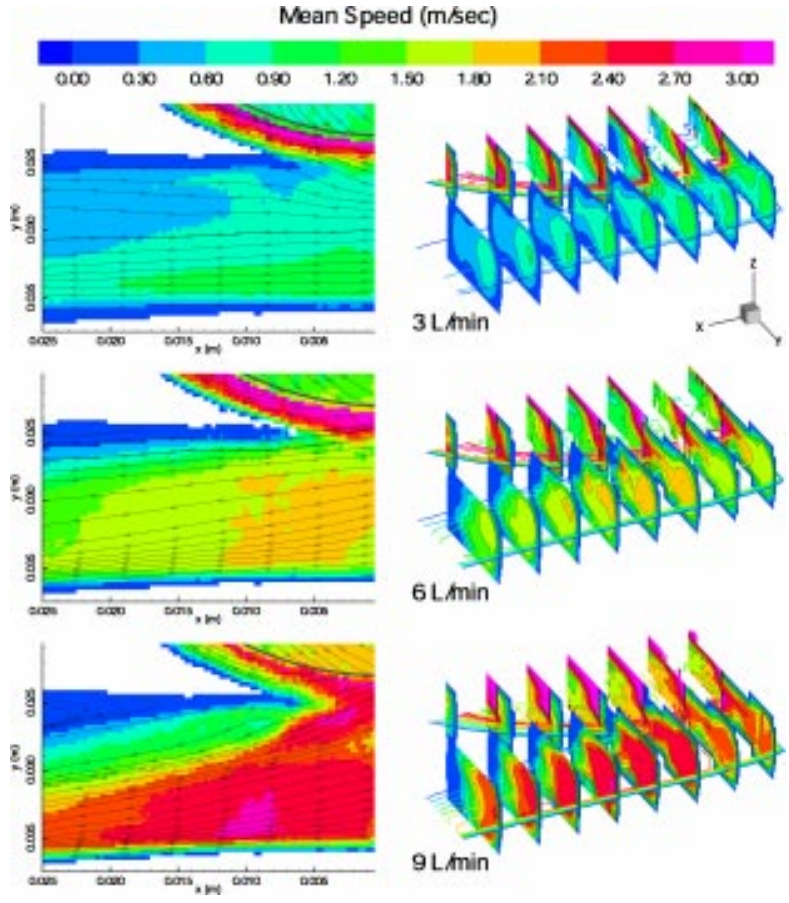

Fig. 8 Distribution of mean speed within the cutwater for flowrates of 3,6, and $9 \mathrm{~L} / \mathrm{min}$. One plane of constant $z$, at cutwater centerline, and several planes of constant $x$ are shown. 


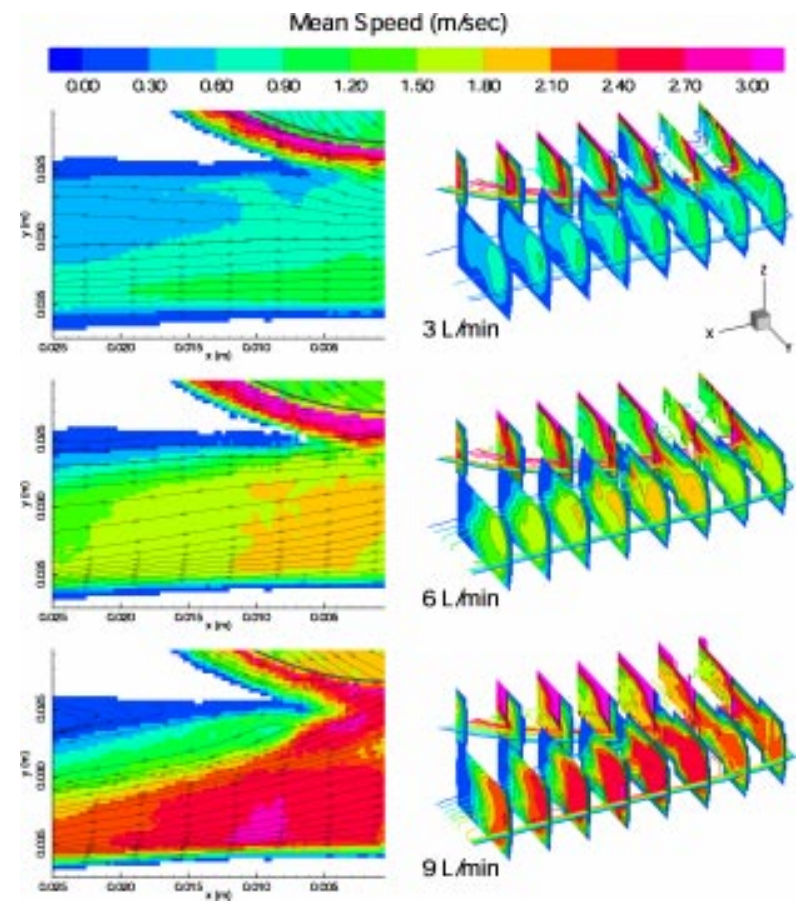

Fig. 9 Distribution of rms of fluctuating speed (left) and MRSS (right) within the cutwater for flow rates of 3,6 , and $9 \mathrm{~L} / \mathrm{min}$ within the cutwater center plane

portional to flow rate, as were the mean flow velocities, so that the rms was nearly a constant $15 \%$ of the mean velocity for all flow rates between 3 and $9 \mathrm{~L} / \mathrm{min}$.

The distribution of MRSS is similar to the distribution of rms, with maximum levels of $300 \mathrm{~Pa}$ in the shear layer at the blade exit during $3 \mathrm{~L} / \mathrm{min}$ and of about $200 \mathrm{~Pa}$ in the cutwater wake at $9 \mathrm{~L} / \mathrm{min}$ (Fig. 9). In contrast to the distribution of rms, levels of MRSS were not everywhere high; with the majority of the region having MRSS levels less than $30 \mathrm{~Pa}$.

As was true for the other regions of the pump, levels of viscous shear are everywhere much lower than Reynolds stresses. Values of viscous shear are most significant along the outer wall of the exit volute, both just outside and inside the cutwater. The magnitude of viscous stresses along the volute wall increases with flow rate, as would be expected, and reaches a maximum $(\sim 12 \mathrm{~Pa})$ at $9 \mathrm{~L} / \mathrm{min}$.

Error Analysis. Traditional PIV with a single camera measures only two components of velocity, and is limited to speeds and turbulence properties based only on the in-plane components of velocity. The accuracy and precision of PIV measurements based on digital images have been studied extensively by numerous investigators $[48,49]$, including techniques to correct or minimize these errors [50,51]. Additionally, there is a continuing effort to develop analysis algorithms for increased accuracy and spatial resolution $[43,52,53]$.

The instantaneous velocity measurement at a single measurement point contains an error that can be decomposed into a systematic error $e_{\text {bias }}$ and a random error $e_{\text {rms }}$ Assuming adequate density of seed particles, uncertainty due to random errors is a function of the interrogation window size and the diameter of an individual particle's images. A particle image diameter of 1-3 pixels and $16 \times 16$ pixel interrogation window (conditions for the current experiment) leads to an $e_{\mathrm{rms}}$ of $0.06-0.08$ pixels [54]. Because of the similarity in density of the particles and fluid and the relatively high viscosity of the fluid, both the settling velocity and transient response of particles to changes in velocity are negligible [54].
The spatial resolution (smallest resolvable scales in the flow) of this technique is approximately equal to the size of the interrogation window [55], which corresponds to approximately 0.5 $\times 0.5 \mathrm{~mm}$ for these experiments. While this reveals the nature of most of the large-scale steady and random structures in the pump, it is significantly larger than the smallest turbulent length scales in the flow.

Due to the low sampling rate $(10 \mathrm{~Hz})$, the technique cannot resolve the frequency spectrum for frequencies higher than $5 \mathrm{~Hz}$ according to Shannon's sampling theorem [56]. At a mean velocity of $1 \mathrm{~m} / \mathrm{s}, 5 \mathrm{~Hz}$ corresponds to $20 \mathrm{~cm}$ structures in the flow, significantly larger than the pump itself. Although the frequency spectrum above $5 \mathrm{~Hz}$ cannot be determined, this portion of the spectrum is aliased and does still contribute to the statistics that are summed over the entire spectrum, such as the mean and rms of fluctuating velocity. The maximum frequency fluctuation to which the technique is sensitive is determined by the time separation of the two images, and is approximately $10^{4} \mathrm{~Hz}(1 / \Delta t$ with $\Delta t$ $=100 \mathrm{~ms}$ ) [55]. Statistical treatment of the fluctuations in the velocity field based on these measurements is sensitive to the frequency range of approximately $0-10^{4} \mathrm{~Hz}$. The integral length scales $L$ and Kolmogorov length scales $\eta$ are typically used to characterize the size of the largest and smallest turbulent structures, respectively. The size of the integral length scales is on the order of the geometry of the pump, 2-12 mm, as estimated from Figs. 4, 7, and 9. At 1-3 m/s, this corresponds to 100-500 Hz and is within the sensitivity range of the technique. The estimated size of the Kolmogorov length scales $\left(2.5 \times 10^{-5}-1 \times 10^{-4} \mathrm{~m}\right.$ based on balancing production and dissipation according to the method described in Tennekes and Lumley [57]) corresponds roughly to a frequency of $1 \times 10^{4}-1 \times 10^{5} \mathrm{~Hz}$, which is as much as one order of magnitude above the sensitivity range of the technique.

Several studies have shown that statistical values of turbulent properties are estimated fairly accurately with PIV, even when the size of the interrogation region is only slightly smaller than the integral length scale of the turbulent fluctuations $[43,58,59]$. Because the majority of turbulent energy is contained in the lowfrequency fluctuations, a statistical characterization of the flow based on measurements in a limited frequency range is effective. It has been shown that spatial resolution of approximately $90 \eta(90$ times the size of the Kolmogorov scales) leads to $65 \%$ of the actual turbulence intensity, and similarly $95 \%$ of the true value of turbulent kinetic energy is achieved with a resolution of $20 \eta$ [59].

\section{Discussion}

Unsteadiness in Inlet Elbow. The large-scale (low-frequency) fluctuations seen in the inlet elbow at constant flow rate may be caused by two effects. First, flow instabilities generated by the inlet cannula or the attachment of the cannula to the ventricle may persist through the length of cannula and into the inlet elbow. Based on the flow rate and inlet diameter, the Reynolds number for the inlet cannula ranges from laminar to transitional (10003000 ) over a range of flow rates of $3-9 \mathrm{~L} / \mathrm{min}$. For the laminar flow case, the cannula length $(200 \mathrm{~mm}=16$ dia $)$ is not long enough to guarantee fully developed flow at the pump. For a straight pipe, an entry length of $375 \mathrm{~mm}$ (30 dia) would insure fully developed flow [60], but is an unrealistic boundary condition for this pump. For the implanted condition, the flow into the pump may be significantly less like developed pipe flow than these test conditions because the inlet catheter will be shorter and more curved. A second possible cause of large-scale fluctuations is that rotation of the impeller induces a swirl in the inlet pipe of the pump [47]. While the effects of this on flow in the inlet pipe and resulting blade incidence angles are known for a straight inlet pipe, they are not as clear for a complex geometry like the inlet elbow of this pump [47]. The observed effects may be the result of the combination of upstream (inlet and entrance effects) and 
downstream (impeller swirl) phenomena. In all other regions of the pump, the fluctuations are significantly smaller in size.

Comparison to Other Studies. Similarly high levels of velocity fluctuations (5-40\% of local mean velocity in this study) at the blade exit or within the volute of a larger pump as measured by LDV have been reported by Flack et al. [10]. A discussion of these results by Cumptsy is also included in the same publication questioning whether the reported turbulence intensities are actually the results of variance in the flow from some cause other than turbulence. In the experiments of Flack and the measurements in this study, variations at the blade pass and impeller rotational frequency were removed from the measured fluctuations before calculating turbulent intensities. The flow rate in both experiments was monitored to eliminate variations in flow rate as the cause. Levels of turbulence intensity reported by Flack within the blade passage, but near the outer diameter of the impeller are $\sim 8 \%$ at the design flow and as high as $25 \%$ at $40 \%$ of the design capacity. This shows both similar magnitudes and the same trend of increased rms of velocity fluctuations at lower than design capacities as is seen in the current measurements in the high shear region of the blade exit.

Reports of measured viscous and Reynolds shear stresses in rotary blood pumps are limited. Asztalos reported viscous shear rates of 9,000-19,000 1/s (viscous stress levels of 27-57 Pa, assuming a viscosity of $\left.3 \times 10^{-3} \mathrm{~N}-\mathrm{s} / \mathrm{m}^{2}\right)$ in the volute region of a rotary pump [28], but did not report Reynolds stresses. This is higher than measured viscous stresses in the volute of this pump $(\sim 15 \mathrm{~Pa})$, but significantly lower than measured Reynolds stresses $(\sim 300 \mathrm{~Pa})$.

Yoganathan et al. [61] reported peak Reynolds stresses between $350-450 \mathrm{~Pa}$ using LDV on 13 aortic valve prostheses. Baldwin et al. [17] reported Reynolds stresses as high as $360 \mathrm{~Pa}$ for forward flow and $550 \mathrm{~Pa}$ for regurgitant flow through a closing disc valve and later showed that the actual peak stresses were as high as 1000 for very short periods of time [19]. Hot-film anemometry has also been used to measure the Reynolds shear stress downstream of prosthetic valves to be as high as $200 \mathrm{~Pa}$ [62]. Overall, the reported Reynolds stresses of 180-450 Pa in valve prostheses correspond to turbulence intensities of approximately $25-50 \%$ at the reported axial velocity $(\sim 1 \mathrm{~m} / \mathrm{sec})$ and are similar to those measured in this pump (maximum $300 \mathrm{~Pa}$ Reynolds stress, 40\% turbulence intensity).

Implications for Blood Damage. The determination of overall qualitative flow patterns is useful in locating regions of pump inefficiency and potential for blood damage, as has been shown in prior studies $[15,21]$. When combined with considerations of measured stress levels, even better estimations of blood damage may be made. Within this pump, levels of viscous stresses are everywhere below $15 \mathrm{~Pa}$, which is one order of magnitude less than the lowest published laminar stress threshold for cell lysis and below those reported for platelet activation $[63,64]$. At the design flow rate $(6 \mathrm{~L} / \mathrm{min})$, the flow is generally well behaved (no recirculation or stagnant flow other than that described in the inlet elbow) and Reynolds shear levels are below levels that would be expected to contribute to hemolysis or thrombosis. However, flow at both high $(9 \mathrm{~L} / \mathrm{min})$ and low $(3 \mathrm{~L} / \mathrm{min})$ flow rates introduces anomalies into the flow such as recirculation, stagnation, and high stress regions that may cause potential problems. These factors were considered in evaluating the flow everywhere within the pump and two regions of the flow path were selected as those with the most potential to cause damage. These are the shear layer at blade exit during low flow conditions and the cutwater separation during high flow conditions. Within both, levels of MRSS, MRNS, and viscous stress are examined here.

At the design and higher flow rates, the velocity gradient at the blade exit is not as pronounced, so stress levels are not very high (Fig. 6). At lower than design flow rates $(3 \mathrm{~L} / \mathrm{min})$, however, a significant gradient exists and is associated with MRSS of
300 Pa. Further, streamlines indicate that a blood particle could stay within the shear layer for an extended period of time. During diastole, the flow rate is low and nearly constant for approximately one half of the heartbeat, so a conservative estimate of the residence time in this high shear region is $0.5 \mathrm{~s}$. The second region of note is the cutwater region. During high flow, significant turbulent stresses (MRSS $\sim 240 \mathrm{~Pa}$ ) and a recirculation region exits, as shown in Fig. 6. A conservative estimate of the residence time in this region is 0.5 seconds, approximately the duration of systole.

While the equation given by Giersiepen et al. [2] would predict $>1 \%$ cell lysis at $300 \mathrm{~Pa}$ for all exposure times greater than 0.01 $\mathrm{s}$, it should be remembered that this equation is a fit to viscous, not turbulent stresses. With the exception of Sutera [65], for which the exposure time was $200 \mathrm{~s}$, the lowest published threshold values of Reynolds stresses is $400 \mathrm{~Pa}$ reported by Sallam and Hwang [66]. Even this has been reported to be an underestimate of the actual value, so that values of $600-800 \mathrm{~Pa}$ are more reasonable $[67,68]$. Considering this, measured Reynolds stresses of $300 \mathrm{~Pa}$ within this pump are not anticipated to induce significant levels of hemolysis. Measurements of hemolysis during animal implant and blood bag tests (unpublished) also indicate that the pump-induced cell lysis is negligible.

Levels of viscous and turbulent shear stress required for platelet activation are significantly lower than for red cell lysis. Previously stated levels of stress for platelet activation due to viscous stresses were as low as $50 \mathrm{~Pa}$ at exposure times of $100 \mathrm{~ms}$ [69]. Even considering that the significant stresses in this pump are Reynolds stresses instead of viscous stresses, it is plausible that Reynolds stresses in the range of $300 \mathrm{~Pa}$ will have some effect on platelet activation.

The sequence of events required to promote thrombosis is summarized by: (i) Flow disturbance activates platelets or lyses some red cells. (ii) Recirculation causes platelet activation to amplify by extended exposure to activated platelets and lysed red cells. (iii) Reattachment and low wall stresses increase the collisions of suspended particles with the wall, allowing platelets to adhere and the formation of a thrombus [69]. The flow at high flow rates in the cutwater and low flow rates within the blade passage could encourage thrombus formation through this sequence of events. In the cutwater wake, flow passes through a high stress region and then some of the blood is entrained into the recirculation region, creating the potential for thrombus formation at or near the reattachment point. Similarly, the recirculation region on the pressure side of the blade has the potential to accumulate thrombus at low flow rates.

While there are not other obvious recirculation regions within the pump that will lead to the formation of attached thrombus, activated platelets may be released into the blood stream. Activated platelets can adhere to biological surfaces downstream of the pump or adhere to one another to form blood-borne microthromboemboli that would be filtered out of the bloodstream by small vessels in the tissue or organs or potentially clog small peripheral arteries.

In summary, significant levels of hemolysis and attached thrombus are not anticipated within the pump. This is particularly true for pulsatile flow, due to the additional blood washing created by time-varying flow fields [34]. Levels of turbulent shear stress are, however, high enough to initiate platelet activation and the flow field provides transient flow structures that could act to amplify the hemostatic reaction. If this does occur, the small thrombus created within the pump may enter the circulatory system with the potential to cause damage to tissues and organs. The strictest design and analysis criteria for blood damage would be to also limit the releasing of activated platelets into the bloodstream.

\section{Conclusions}

The measurements from this work show that PIV is an extremely useful aid to the design process because it provides both 
qualitative characteristics of the flow field and quantitative measurements of speed and stress levels within the pump. The impeller of the prototype used in these experiments was mounted on a shaft instead of suspended by magnetic bearings. Prior to these experiments, a comparison of computational fluid dynamics (CFD) simulations of the flow in the back clearance gap with and without the rotating shaft and teeth indicated that the shaft and teeth did not affect the flow in the back clearance or in other regions of the pump. Because of the measurements reported here, and earlier flow visualization experiments of recirculation in the inlet elbow and the separation and recirculation zone in the cutwater (both CF4b), the geometry of the inlet and cutwater were modified in the subsequent iteration of this pump (CF4c). Additionally, quantitative measurements are essential for the validation and refinement of computational models that are commonly used in the design of these devices.

The design evolution of artificial heart valves is an example of a technology that is more mature than rotary LVADs and has successfully integrated basic numerical, theoretical, and experimental fluid dynamics into the design process in order to create more effective devices [70,71]. These studies have demonstrated several relevant issues. First, regions of high shear stress caused by small clearances in the valves can lead to unacceptable levels of hemolysis [72,73]. Second, turbulent regions of recirculation and stagnation in the flowfield, even if they are only present for part of the pulse cycle, can lead to thrombus formation $[74,75]$. Third, computational models of the flow field need to be validated and refined with experimental measurements in order to accurately reproduce the necessary details of the flow [76,77]. Finally, design iterations guided by experimental measurements and numerical models can be used to drastically reduce blood damage caused by the high shear stress and recirculation regions surrounding the valves. It is hoped that the current study will contribute to the similar progression in understanding of rotary ventricular assist device fluid mechanics and design that is currently underway.

While the measurements presented herein are for one particular pump, they have some applicability to other rotary LVADs. Specifically, turbulent flow and associated Reynolds stresses are unavoidable, considering required flow rates and relatively small dimensions of an implantable pump. Regions of flow stagnation are unavoidable at the flow cutwater. The high shear region at blade exit is caused by the velocity gradient between fluid exiting the impeller and flow in the exit volute. A sufficiently high absolute velocity at the impeller exit is required to generate the required pressure rise across the pump, so it is expected that similarly high stresses will exist in the shear layers of other pumps. Lastly, the problem of requiring a constant-speed rotary pump to operate over a wide range of flow rates is common to all rotary LVADs that are attached between the left ventricle and aorta. It is essential that the design of the fluid pathway within rotary LVADs must consider flow rates other than the nominal operating point because of this.

\section{Acknowledgments}

Financial support for this work has been provided by the Utah Artificial Heart Institute and the Department of Health and Human Services, National Institutes of Health, National Heart, Lung, and Blood Institute Grant No. R01 HL64378-01. The authors would also like to thank F. Scarano and M. L. Reithmuller for use of their PIV algorithm, the Artificial Heart Center at UVa, Phil Lemire for help with the test rig, and MedQuest ${ }^{\circledR}$ Products for the fabrication of the prototype pump. Comments from the anonymous reviewers were extremely helpful in revising the original manuscript.

\section{Nomenclature}

$$
\begin{aligned}
\Delta t & =\text { time separation between acquired images } \\
\tilde{u} & =\text { instantaneous velocity }
\end{aligned}
$$

$$
\begin{aligned}
U & =\text { time averaged mean velocity } \\
u & =\text { fluctuating component of velocity } \\
u^{\prime} & =\text { rms fluctuating velocity, } u \\
N & =\text { number of instantaneous measurements in a } \\
& \text { series } \\
\text { MRNS } & =\text { major Reynolds normal stress } \\
\text { MRSS } & =\text { major Reynolds shear stress } \\
\mu & =\text { absolute or dynamic fluid viscosity }
\end{aligned}
$$

\section{References}

[1] Heuser, G., and Opitz, R., 1980, "A Couette Viscometer for Short Time Shearing of Blood," Biorheology 17(2), pp. 17-24.

[2] Giersiepen, M., Wurzinger, L. J., Optiz, R., and Reul, H., 1990, "Estimation of Shear Stress Related Blood Damage in Heart Valve Prostheses: In Vitro Comparison of 25 Aortic Valves," Int. J. Artif. Organs 13(5), pp. 300-306.

[3] Goldsmith, H. L., and Turitto, V. T., 1986, "Rheological Aspects of Thrombosis and Haemostasis: Basic Principles and Applications," Thromb. Haemostasis $\mathbf{5 5}(3)$, pp. $415-435$.

[4] Berger, S. A., and Jou, L.-D., 2000, "Flows in Stenotic Vessels," Annu. Rev. Fluid Mech. 32, pp. 347-382.

[5] Copeland, J. G., 1987, "The Artificial Heart as a Bridge to Transplant," Cardio, Oct., pp. 44-47.

[6] Brownell, R., Flack, R., and Kostrowsky, G., 1985, "Flow Visualization in the Tongue Region of a Centrifugal Pump," J. Therm. Eng. 4(2), pp. 35-45.

[7] Akhras, A. R., El Hajem, M., Morel, R., and Champagne, J. Y., 2001, "Internal Flow Investigation of a Centrifugal Pump at the Design Point," J. Visualization 4(1), pp. 91-98.

[8] Hamkins, C., and Flack, R., 1987, "Laser Velocimeter Measurements in Shrouded and Unshrouded Radial Flow Pump Impellers," ASME J. Turbomach. 109(1), pp. 70-76.

[9] Miner, S. M., Beaudoin, R. J., and Flack, R. D., 1989, "Laser Velocimetry Measurements in a Centrifugal Flow Pump," ASME J. Turbomach. 111(3), pp. 205-212.

[10] Flack, R. D., Miner, S. M., and Beaudoin, R. J., 1992, "Turbulence Measurements in a Centrifugal Pump With a Synchronously Orbiting Impeller," ASME J. Turbomach. 114, pp. 350-359.

[11] Ojeda, W. D., Flack, R. D., and Miner, S. M., 1995, "Laser Velocimetry Measurements in a Double Volute Centrifugal Pump," Int. J. Rotating Mach. 1(34), pp. 199-214.

[12] Rajendran, V., and Patel, V., 2000, "Measurement of Vortices in Model PumpIntake Bay by PIV," J. Hydraul. Eng. 126(5), pp. 322-334.

[13] Dong, R. , Chu, S., and Katz, J. S., 1992, "Quantitative Visualization of the Flow Within the Volute of a Centrifugal Pump. Part A: Technique," ASME J. Fluids Eng. 114(3), pp. 390-395.

[14] Dong, R. , Chu, S., and Katz, J. S., 1997, "Effect of Modification to Tongue and Impeller Geometry on Unsteady Flow, Pressure Fluctuations, and Noise in a Centrifugal Pump," ASME J. Turbomach. 119(3), pp. 506-515.

[15] Orime, Y., et al., 1994, "The Baylor Total Artificial Heart," ASAIO J. 40, pp. M499-M505.

[16] Rose, M. L. J., Mackay, T. G., and Wheatley, D. J., 2000, "Evaluation of Four Blood Pump Geometries: Fluorescent Particle Flow Visualisation Technique," Med. Eng. Phys. 22(3), pp. 201-214.

[17] Baldwin, J. T., Deutsch, S., Geselowitz, D. B., and Tarbell, J. M., 1994, “LDA Measurements of Mean Velocity and Reynolds Stress-Fields Within an Artificial-Heart Ventricle," ASME J. Biomech. Eng. 116(2), pp. 190-200.

[18] Mussivand, T. , Day, K. D., and Naber, B. C., 1999, "Fluid Dynamic Optimization of a Ventricular Assist Device Using Particle Image Velocimetry," ASAIO J. 45(1), pp. 25-31.

[19] Maymir, J. C., Deutsch, S., Meyer, R. S., Geselowitz, D. B., and Tarbell, J. M., 1998, "Mean Velocity and Reynolds Stress Measurements in the Regurgitant Jets of Tilting Disk Heart Valves in an Artificial Heart Environment," Ann. Biomed. Eng. 26(1), pp. 146-156.

[20] Nishida, M., et al., 2000, "Effect of Washout Hole Geometry on a Centrifugal Blood Pump," ASAIO J. 46(2), pp. 172-178.

[21] Araki, K., et al., 1993, "A Flow Visualization Study of Centrifugal Blood Pumps Developed for Long-Term Usage,” Artif. Organs 17(5), pp. 307-312.

[22] Asztalos, B., et al., 1996, "Flow Visualization Study of Centrifugal Blood Pump for Total Artificial Heart," Proc. 18th Annual International Conference of the IEEE Engineering in Medicine and Biology Society, IEEE, New York, pp. 1347-1348.

[23] Ng, B. T. H., Chan, W. K., Yu, S. C. M., and Li, H. D., 2000, "Experimenta and Computational Studies of the Relative Flow Field in a Centrifugal Blood Pump," Crit. Rev. Biomed. Eng. 28(1-2), pp. 119-125.

[24] Apel, J., Neudel, F., and Reul, H., 2001, "Computational Fluid Dynamics and Experimental Validation of a Microaxial Blood Pump," ASAIO J. 47, pp. 552-558.

[25] Baldwin, J., Tarbell, J., Deutsch, S., and Geselowitz, D., 1989, "Mean Flow Velocity Patterns Within a Ventricular Assist Device," ASAIO Trans. 35(3), pp. 429-433.

[26] Manning, K. B., and Miller, G. E., 2002, "Flow Through an Outlet Cannula of a Rotary Ventricular Assist Device," Artif. Organs 26(8), pp. 714-723.

[27] Day, S. W., et al., 2001, "Particle Image Velocimetry Measurements of Blood Velocity in a Continuous Flow Ventricular Assist Device," ASAIO J. 47(4), 
pp. 406-411.

[28] Asztalos, B., Yamane, T., and Nishida, M., 1999, "Flow Visualization Analysis for Evaluation of Shear and Recirculation in a New Closed-Type, Monopivot Centrifugal Pump," Artif. Organs 23(10), pp. 939-946.

[29] Bearnson, G., et al., 2000, "Progress on the Heartquest VAD - A Centrifugal Pump With Magnetically Suspended Rotor," ASAIO J. 46(2), p. 192.

[30] Song, X., Wood, H. G., and Olsen, D., 2003, "Computational Fluid Dynamics (CFD) Study of the 4th Generation Prototype of a Continuous Flow Ventricular Assist Device (VAD),” ASME J. Biomech. Eng. 126(2), pp. 180-187.

[31] Day, S. W., et al., 2002, "A Prototype HeartQuest Ventricular Assist Device for Particle Image Velocimetry Measurements," Artif. Organs 26(11), pp. 10021005.

[32] Day, S. W., Lemire, P. P., Flack, R. D., and McDaniel, J. C., 2003, "Effect of Reynolds Number on Performance of a Small Centrifugal Pump," Proc. 4th ASME/JSME Joint Fluids Engineering Conference, Hawaii, ASME, New York.

[33] Akimoto, T., et al., 1999, "Rotary Blood Pump Flow Spontaneously Increases During Exercise Under Constant Pump Speed - Results of a Chronic Study," Artif. Organs 23(8), pp. 797-801.

[34] Day, S. W., and McDaniel, J. C., "PIV Measurements of Flow in a Centrifugal Blood Pump: Time-Varying Flow," ASME J. Biomech. Eng. (in press).

[35] Adrian, R. J., 1991, "Particle Imaging Techniques for Fluid Mechanics," Annu. Rev. Fluid Mech. 23, pp. 261-304.

[36] Keane, R. D., and Adrian, R. J., 1992, "Theory of Cross-Correlation Analysis of PIV Images,” Appl. Sci. Res. 49, pp. 191-215.

[37] Durst, F., Melling, A., and Whitelaw, J., 1976, Principles and Practice of Laser Doppler Anemometry, Academic Press, New York.

[38] Baldwin, J. T., Deutsch, S., Petrie, H. L., and Tarbell, J. M., 1993, "Determination of Principal Reynolds Stresses in Pulsatile Flows After Elliptical Filtering of Discrete Velocity Measurements," ASME J. Biomech. Eng. 115(4), pp. 396-403.

[39] Narrow, T. L., Yoda, M., and Abdel-Khalik, S. I., 2000, “A Simple Model for the Refractive Index of Sodium Iodide Aqueous Solutions,” Exp. Fluids 28, pp. 282-283.

[40] Durst, F., Muller, R., and Jovanovic, J., 1988, "Determination of the Measuring Position in Laser-Doppler Anemometry," Exp. Fluids 6(2), pp. 105-110.

[41] Budwig, R., 1994, "Refractive Index Matching Methods for Liquid Flow Investigations," Exp. Fluids 17, pp. 350-355.

[42] Wells, R. E., and Merrill, E. W., 1961, "Shear Rate Dependence of the Viscosity of Whole Blood and Plasma," Science 133, pp. 763-764.

[43] Scarano, F., and Reithmuller, M., 1999, "Iterative Multigrid Approach in PIV Image Processing With Discrete Window Offset," Exp. Fluids 26, pp. 513523

[44] Wernet, M., 2000, "Application of DPIV to Study Both Steady State and Transient Turbomachinery Flows," Opt. Laser Technol. 32, pp. 497-525.

[45] Bendat, J. S., and Piersol, A. G., 1971, Random Data: Analysis and Measurement Procedures, Wiley Interscience, New York.

[46] Day, S. W., 2003, "Measurements of Flow in a Centrifugal Blood Pump Using Particle Image Velocimetry," Ph.D. thesis, University of Virginia, Charlottesville.

[47] Stepanoff, A. J., 1957, Centrifugal and Axial Flow Pumps, Wiley, New York.

[48] Huang, H., Dabiri, D., and Gharib, M., 1997, "On Errors of Digital Particle Image Velocimetry," Meas. Sci. Technol. 8(12), pp. 1427-1440.

[49] Adrian, R. J., 1997, "Dynamic Ranges and Spatial Resolution of Particle Image Velocimetry," Meas. Sci. Technol. 8(12), pp. 1393-1398.

[50] Boillot, A., and Prasad, A. K., 1996, "Optimization Procedure for Pulse Separation in Cross-Correlation PIV," Exp. Fluids 21, pp. 81-93.

[51] Hart, D. P., 2000, “PIV Error Correction,” Exp. Fluids 29, pp. 13-22.

[52] Cowen, E., and Monismith, S., 1997, "A Hybrid Digital Particle Tracking Velocimetry Technique," Exp. Fluids 22, pp. 199-211.

[53] Lourenco, L. M., and Krothapalli, A., 2000, "TRUE Resolution PIV: A MeshFree Second-Order Accurate Algorithm," Proc. 10th International Symposium of Laser Techniques in Fluid Mechanics, Lisbon, Portugal, paper 13.5.

[54] Raffel, M., Willert, C., and Kompenhans, J., 1998, Particle Image Velocimetry: A Practical Guide, Springer, New York.
[55] Adrian, R. J., 1988, "Statistical Properties of Particle Image Velocimetry Measurements in Turbulent Flow," Proc. Laser Anemometry in Fluid Dynamics III, Lisbon, Portugal, pp. 115-129.

[56] Shannon, C. E., 1949, "Communication in the Presence of Noise," Proc. Inst Radio Eng. 37(1), pp. 10-21.

[57] Tennekes, H., and Lumley, J. L., 1972, A First Course in Turbulence, MIT, Cambridge, MA

[58] Lecordier, B., Demare, D., Vervisch, L. M. J., Reveillon, J., and Trinite, M., 2001, "Estimation of the Accuracy of PIV Treatments for Turbulent Flow Studies by Direct Numerical Simulation of Multi-Phase Flow,” Meas. Sci. Technol. 12, pp. 1382-1391.

[59] Saarenrinne, P., Piirto, M., and Eloranta, H., 2001, "Experience of Turbulence Measurement With PIV," Meas. Sci. Technol. 12, pp. 1904-1910.

[60] Fox, R. W., and McDonald, A. T., 1992, Introduction to Fluid Mechanics, Wiley, New York.

[61] Yoganathan, A. P., Woo, Y. R., and Sung, H. W., 1986, "Turbulent Shear Stress Measurements in the Vicinity of Aortic Heart Valve Protheses," J. Biomech. 19, pp. 422-433.

[62] Nygaard, H., et al., 1990, "Estimation of Turbulent Shear Stress in Pulsatile Flow Immediately Downstream of Two Artificial Aortic Valves in vitro," J. Biomech. 23(12), pp. 1231-1238.

[63] Leverett, L. B., Hellums, J. D., Alfrey, C. P., and Lynch, E. C. U., 1972, "Red Blood Cell Damage by Shear Stress," Biophys. J. 12(3), pp. 257-273.

[64] Wurzinger, L. J., Blasberg, P., and Schmid-Schonbein, H. U., 1985, “Towards a Concept of Thrombosis in Accelerated Flow: Rheology, Fluid Dynamics, and Biochemistry," Biorheology 22(5), pp. 437-450.

[65] Sutera, S. P., and Mehrjardi, M. H., 1975, "Deformation and Fragmentation of Human Red Blood Cells in Turbulent Shear Flow," Biophys. J. 15, pp. 1-10.

[66] Sallam, A. M., and Hwang, N. H. C., 1984, "Human Red Cell Hemolysis in Turbulent Shear Flow: Contribution of Reynolds Shear Stresses," Biorheology 21, pp. 783-797.

[67] Grigioni, M., Daniele, C., D’Avenio, G., and Barbaro, V., 1999, “A Discussion on the Threshold Limit for Hemolyis Related to Reynolds Shear Stress," J. Biomech. 32(10), pp. 1107-1112.

[68] Lu, P. C., Lai, H. C., and Liu, J. S., 2001, "A Reevaluation and Discussion on the Threshold Limit for Hemolysis in a Turbulent Shear Flow," J. Biomech. 34(10), p. 1364.

[69] Wurzinger, L. J., and Schimid-Schoenbein, H., 1990, "The Role of Fluid Dynamics in Triggering and Amplifying Hemostatic Reactions in Thrombogenesis," Blood Flow in Large Arteries: Applications to Atherogenesis and Clinical Medicine, D. W. Liepsch, ed., Karger, New York, pp. 215-226.

[70] Peskin, C. S., 1982, "The Fluid Dynamics of Heart Valves: Experimental, Theoretical, and Computational Methods," Annu. Rev. Fluid Mech. 14, pp. 235-259.

[71] Healy, T. M., Fontaine, A. A., Walton, S. P., and Yoganathan, A. P., 1998 "Visualization of the Hinge Flow in a 5:1 Scaled Model of the Medtronic Parallel Bileaflet Heart Valve Prothesis," Exp. Fluids 25(5/6), pp. 512-518.

[72] Baldwin, J. T., Tarbell, J. M., Deutsch, S., and Geselowitz, D. B., 1991, "Mean Velocities and Reynolds Stresses Within Regurgitant Jets Produced by Tilting Disc Valves," ASAIO Trans. 37(3), pp. M348-M349.

[73] Schoephoerster, R. T., and Chandran, K., 1991, "Velocity and Turbulence Measurements Past Mitral Valve Prostheses in a Model Left Ventricle," J. Biomech. 24(7), pp. 549-562.

[74] Mandrusov, E., Puszkin, E., Vroman, L., and Leonard, E., 1996, "Separated Flows in Artificial Organs: A Cause of Early Thrombogenesis?," ASAIO J. 42(5), pp. 506-513.

[75] Travis, B. R., et al., 2001, "Bileaflet Aortic Valve Prosthesis Pivot Geometry Influences Platelet Secretion and Anionic Phospholipid Exposure," Ann. Biomed. Eng. 29, pp. 657-664.

[76] Krafczyk, M., Cerrolaza, M., Schulz, M., and Rank, E., 1998, "Analysis of 3D Transient Blood Flow Passing Through an Artificial Aortic Valve by LatticeBoltzmann Methods," J. Biomech. 31(5), pp. 453-462.

[77] Aluri, S., and Chandran, K., 2001, "Numerical Simulation of Mechanical Mitral Heart Valve Closure," Ann. Biomed. Eng. 29(8), pp. 665-676. 